\title{
AN ANALYTICAL SOLUTION FOR THE BLACK-SCHOLES EQUATION USING FUNCTIONAL PERTURBATION METHOD
}

\author{
SOMAYEH POURGHANBAR, MOJTABA RANJBAR*, AND EBRAHIM NASRABADI
}

\begin{abstract}
One of the greatest accomplishments in modern financial theory, in terms of both approach and applicability has been the Black-Scholes option pricing model. It is widely recognized that the value of a European option can be obtained by solving the BlackScholes equation. In this paper we use functional perturbation method (FPM) for solving Black-Scholes equation to price a European call option. The FPM is a tool based on considering the differential operator as a functional. The equation is expanded functionally by Frechet series. Then a number of successive partial differential equations (PDEs) are obtained that have constant coefficients and differ only in their right hand side part. Therefore we do not need to resolve the different equations for each step. In contrast to methods that have implicit solutions, the FPM yields a closed form explicit solution.
\end{abstract}

Keywords: Black-Scholes equation; European call option; Functional perturbation method.

\section{Introduction}

An option is a financial contract whose value is dependent on the price of a stock [14]. An option gives its owner the right to buy or sell a specified amount of a particular asset at a fixed price, on or before a specified date [23]. Options can be grouped into either of the two categories: European options and American options. As said in [24], options can be either call or put, a call option gives its holder the right to buy a prescribed asset at a prescribed price (exercise price), while a put option gives its holder the right to sell a prescribed asset at exercise price. Following the seminal work of Black and Scholes [10] the pricing of stock options has become increasingly desirable in the economic and finance literature. Classical asset pricing theory assumes that traders act as price takers [22]. In parallel with this theory, most of the option pricing models have been done in liquid markets. According to the BlackScholes model, the price of an option's underlying asset $x_{t}$, follows a geometric Brownian motion [17] ( $x_{t}$ satisfies the following stochastic differential equation)

$$
d x_{t}=\mu x_{t} d t+\sigma x_{t} d B_{t},
$$

In which $\mu$ is the instantaneous expected total return of the stock, $\sigma$ is the instantaneous standard deviation of stock price returns, called the volatility, and $B_{t}$ is the Brownian motion process. The volatility $\sigma$ is IV (implied volatility) or HV (historical volatility). An IV is derived from the market price of a market traded derivative and $\mathrm{HV}$ is derived from time

Date: Received: February 27, 2020, Accepted: March 28, 2020.

${ }^{*}$ Corresponding author. 
series of past market prices. Much research has been devoted to modeling and forecasting the volatility of financial returns. For example Boyle-Vorst model is derived from a binomial model for volatility [11]. Avellaneda and Paras [7] derived a modified volatility model using the algorithm of Bensaid et al. [8]. In addition, we refer the interested reader to [18] for different types of volatility. The Black-Scholes approach makes a number of assumptions about markets like frictionless and completeness [12]. In this situation, the price of a European call option is governed with:

$$
\frac{\partial u(x, t)}{\partial t}+\frac{\sigma^{2}}{2} x^{2} \frac{\partial^{2} u(x, t)}{\partial x^{2}}+r x \frac{\partial u(x, t)}{\partial x}-r u(x, t)=0
$$

with the following conditions

$$
\begin{gathered}
u(0, t)=0, \quad t<T, \\
u(x, t) \sim x, \quad \text { as } \quad x \rightarrow \infty,
\end{gathered}
$$

and

$$
u(x, T)=\max \{x-K, 0\},
$$

where $x$ is the price of the underlying asset at time $t$ and $K$ is exercise price.

Functional perturbation method (FPM) $[4,13,5,16]$ is a powerful mathematical tool for analytical solution of the problems. However, to the best of our knowledge, less attention has been paid to this method in PDEs literature in spite of its applied nature. Perturbation methods using energy principle and shape functions have been studied by Ramu and Ganesan [19]. In a pioneering work, Altus [1] discussed the FPM for elastic problems by replacing an algebraic equation with instead of the original differential equation. As said in [5], perturbation methods have been developed by transforming the equations to an integral form and using projection operators. In [4], the FPM is generalized to treat buckling problems and is compared with the stochastic finite element (SFE) and Monte Carlo Simulation (MCS), and is described the advantages of the FPM. The general problem of finding the best choice for zero order approximation is a fundamental title in optimization problems. Tory et al. [20] applied the FPM to the BDE by operating directly without assuming a restricted set of shape functions. Altus et al. [6] developed an optimized functional perturbation method (OFPM), which is based on finding the best function around which the second order term in the Frechet series vanishes. As showed in [6] OFPM provides more accurate solutions. In [2] the FPM has been used for calculating the probabilistic response of stochastical heterogeneousness. The FPM yields the functional derivatives which are general. As said in [2] this is not just a semantic difference, because in stochastic problems, Frechet series yield an explicit solution. Nachum and Altus [16] applied the FPM for several examples with different non-homogeneous properties. Solutions were obtained by direct integration without resolving the differential equation in [16]. An improvement of FPM is achieved in [13]. Tory et al. [21] applied the FPM directly to the buckling differential equation (BDE). They expanded BDE functionally, yielding a set of ordinary differential equations for each order of the functional derivatives. Furthermore, the optimized DFPM (ODFPM) is introduced by requiring that the second order perturbation term in the Frechet series is minimized.

In this paper the Black-Scholes equation is expanded functionally by Frechet series. Next, a number of PDEs which have constant coefficients are obtained. Moreover, the solution of the Black-Scholes equation as the price of European call option is obtained by FPM.

In the following sections of the article, you can find some necessary preliminaries (Section 2), 
functional perturbation method and its theoretical aspects (Section 3), FPM applicability for Black-Scholes equation (Section 4), and summary of major conclusions (Section 5).

\section{Preliminaries}

We begin our exposition by explaining some basic notations and definitions related to functional algebra. Consider $u$ as a functional of $E$, which is a function of $x$, its derivative is defined by using a Dirac delta function [9]

$$
\frac{\delta u(E)}{\delta E}=\lim _{\eta \rightarrow 0, \epsilon \rightarrow 0} \frac{1}{\epsilon}\left(u\left(E(x)+\epsilon \delta_{\eta}\left(x-x_{0}\right)\right)-u\left(E\left(x_{0}\right)\right)\right)
$$

where $\delta_{\eta}\left(x-x_{0}\right)$ is a function which tends to the Dirac delta function as $\eta \rightarrow 0$. Hereafter, we shall employ the following notation for functional derivative:

$$
\frac{\delta u(E)}{\delta E} \equiv u_{, E}
$$

The Dirac function definition is by the self-functional derivative: (by considering $u(E)=E$ )

$$
u_{, E}=E_{, E}=\delta\left(x-x_{0}\right) .
$$

As said in Appendix of [3], two types of products are useful: inner product and inner integral product (convolution). Here, we use the first for summation and the second for integration:

$$
\sum_{k=1}^{n} u_{k}=u_{k} .1_{k}
$$

where $1_{k}$ are vector elements of unit size, and

$$
\int_{0}^{1} u_{x} d x=u_{x} * 1=1 * u_{x}
$$

where 1 is a unit function. Consistent with this notations, for any given function $E(x)$ we have

$$
u_{, E} * E=\int u_{, E} E(x) d x
$$

and

$$
E * 1=\int_{0}^{1} E(x) d x \equiv\langle E\rangle .
$$

Moreover, perturbation function $E^{\prime}(x)$ is

$$
E^{\prime}(x)=E(x)-\langle E\rangle .
$$

The Dirac function has an important property which we need in this paper:

$$
E\left(x_{0}\right)=\int \delta\left(x-x_{0}\right) E(x) d x .
$$

Therefore, as said in [15], for the sake of convenience, we have

$$
E=\delta * E .
$$


It is worthwhile to know when $E(x)$ is a sufficiently smooth function, (2.9) and (2.10) are called the sifting property or reproducing property of the Dirac function. Besides, according to Ref.[15], an indispensable relation is:

$$
\delta * \frac{\partial E}{\partial x_{i}}=\frac{\partial \delta}{\partial x_{i}} * E=\frac{\partial E}{\partial x_{i}} .
$$

(2.11) can be extended to a differential operator $L$ of each order $p$ as:

$$
\delta * L(E)=(L \delta) * E=L(E) .
$$

A Frechet expansion [5] around the $\langle E\rangle$ is written as:

$$
u(E(x))=u(\langle E\rangle)+u_{, E_{1}} * E_{1}^{\prime}+\frac{1}{2} u_{, E_{1} E_{2}} * * E_{1}^{\prime} E_{2}^{\prime}+\cdots .
$$

All functional derivatives in (2.13) are taken at $\langle E\rangle$. We follow [5] using the notations $u^{(0)}$, $u^{(1)}, u^{(2)}$, etc. as follows:

$$
u(\langle E\rangle) \equiv u^{(0)}, \quad u_{, E_{1}} * E_{1}^{\prime} \equiv u^{(1)}, \quad u_{, E_{1} E_{2}} * * E_{1}^{\prime} E_{2}^{\prime} \equiv u^{(2)} .
$$

\section{Functional Perturbation Method}

To illustrate the basic concepts of the functional perturbation method we consider the following linear differential operator:

$$
L(u)=\phi_{(0)} u+\phi_{(1)} u_{, x}+\phi_{(2)} u_{, x x}+\cdots,
$$

$E=E(x)$ is a given function, $\phi_{(i)}$ is a given set which depend on $E(x)$ or derivatives of $E(x)$ and $u(x)$ is the unknown function. We denote (3.1) as:

$$
L(u)=\phi_{(k)} \cdot u_{, x^{k}},
$$

and we consider $u$ as a functional of $E[3]$ :

$$
u=u(E(x)) .
$$

For special case $E=\langle E\rangle$, Eq. (3.2) will be:

$$
L^{(0)}(u)=\phi_{(k)}^{(0)} \cdot u_{, x^{k}}^{(0)},
$$

where $u_{, x^{k}}^{(0)}=u_{, x^{k}}(\langle E\rangle)$ and $\phi_{(k)}^{(0)}=\phi_{(k)}(\langle E\rangle)$. We have the Frechet expansion for $\phi$ and $L$ as

$$
\begin{gathered}
\phi=\phi(\langle E\rangle)+\phi_{, E_{1}} * E_{1}^{\prime}+\frac{1}{2} \phi_{, E_{1} E_{2}} * * E_{1}^{\prime} E_{2}^{\prime}+\cdots, \\
L(u)=L(u(\langle E\rangle))+L_{, E_{1}} * E_{1}^{\prime}+\frac{1}{2} L_{, E_{1} E_{2}} * * E_{1}^{\prime} E_{2}^{\prime}+\cdots .
\end{gathered}
$$

Considering

$$
\phi(\langle E\rangle) \equiv \phi^{(0)}, \quad \phi_{, E_{1}} * E_{1}^{\prime} \equiv \phi^{(1)}, \quad \phi_{, E_{1} E_{2}} * * E_{1}^{\prime} E_{2}^{\prime} \equiv \phi^{(2)}
$$

and

$$
L(u(\langle E\rangle)) \equiv L^{(0)}, \quad L_{, E_{1}} * E_{1}^{\prime} \equiv L^{(1)}, \quad L_{, E_{1} E_{2}} * * E_{1}^{\prime} E_{2}^{\prime} \equiv L^{(2)} .
$$

Eqs. (3.5) and (3.6) will be

$$
\phi=\phi^{(0)}+\phi^{(1)}+\frac{1}{2} \phi^{(2)}+\cdots,
$$




$$
L(u)=L^{(0)}+L^{(1)}+\frac{1}{2} L^{(2)}+\cdots .
$$

As is stated in [2], since Eq. (3.6) must hold for any $E^{\prime}(x)$, so:

$$
\begin{gathered}
L_{,\left.E_{1}\right|_{\langle E\rangle}}=0, \\
L_{,\left.E_{1} E_{2}\right|_{\langle E\rangle}}=0 .
\end{gathered}
$$

The derivation of (3.2) is:

$$
L_{, E_{1}}=\phi_{(k), E_{1}} u_{, x^{k}}+\phi_{(k)} u_{, x^{k} E_{1}} .
$$

For $E=\langle E\rangle$, we denote:

$$
\left.u_{, x^{k}}\right|_{E=\langle E\rangle}=\left(u^{(0)}\right)_{, x^{k}} \equiv u_{, x^{k}}^{(0)} .
$$

From Eq. (3.11), it is seen that:

$$
L^{(1)}=\left.L_{, E_{1}}\right|_{\langle E\rangle} * E_{1}^{\prime}=\left.\left(\phi_{(k), E_{1}} u_{, x^{k}}+\phi_{(k)} u_{, x^{k} E_{1}}\right)\right|_{\langle E\rangle} * E_{1}^{\prime}=\phi_{(k)}^{(1)} \cdot u_{, x^{k}}^{(0)}+\phi_{(k)}^{(0)} \cdot u_{, x^{k}}^{(1)}=0
$$

where $u^{(0)}$ is known by solving (3.4) and then $u^{(1)}$ can be obtained by solving Eq. (3.15). Because of fulfilling the boundary conditions of the problem by $u^{(0)}$, we consider homogeneous boundary conditions for $u^{(1)}$ and next steps. Now, by Eq. (3.12):

$$
L_{x, E_{1} E_{2}}=\phi_{(i), E_{1} E_{2}} u_{, x^{i}}+\phi_{(i), E_{1}} \cdot u_{, x^{i} E_{2}}+\phi_{(i), E_{2}} \cdot u_{, x^{i} E_{1}}+\phi_{(i)} \cdot u_{, x^{i} E_{1} E_{2}}=0 .
$$

Then

$$
\begin{aligned}
L_{x, E_{1} E_{2}} * * E_{1}^{\prime} E_{2}^{\prime} & =\phi_{(i), E_{1} E_{2}} u_{, x^{i}} * * E_{1}^{\prime} E_{2}^{\prime}+\phi_{(i), E_{1}} \cdot u_{, x^{i} E_{2}} * * E_{1}^{\prime} E_{2}^{\prime} \\
& +\phi_{(i), E_{2}} \cdot u_{, x^{i} E_{1}} * * E_{1}^{\prime} E_{2}^{\prime}+\phi_{(i)} \cdot u_{, x^{i} E_{1} E_{2}} * * E_{1}^{\prime} E_{2}^{\prime} \\
& =\phi_{(i), E_{1} E_{2}} u_{, x^{i}} * * E_{1}^{\prime} E_{2}^{\prime}+\phi_{(i), E_{1}} * E_{1}^{\prime} \cdot u_{, x^{i} E_{2}} * E_{2}^{\prime} \\
& +\phi_{(i), E_{2}} * E_{2}^{\prime} \cdot u_{, x^{i} E_{1}} * E_{1}^{\prime}+\phi_{(i)} \cdot u_{, x^{i} E_{1} E_{2}} * * E_{1}^{\prime} E_{2}^{\prime} \\
& =0 .
\end{aligned}
$$

Also

$$
\phi_{(i)}^{(1)} \equiv \phi_{(i), E_{1}} * E_{1}^{\prime}=\phi_{(i), E_{2}} * E_{2}^{\prime}, \quad u_{, x^{i}}^{(1)} \equiv u_{, x^{i} E_{1}} * E_{1}^{\prime}=u_{, x^{i} E_{2}} * E_{2}^{\prime}
$$

and

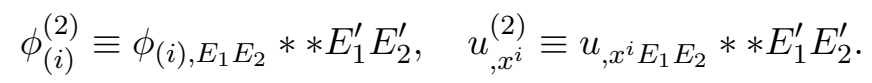

By using (3.18) and (3.19), we can write Eq. (3.17) as:

$$
\phi_{(i)}^{(2)} \cdot u_{, x^{i}}^{(0)}+2 \phi_{(i)}^{(1)} \cdot u_{, x^{i}}^{(1)}+\phi_{(i)}^{(0)} \cdot u_{, x^{i}}^{(2)}=0 .
$$

$u^{(2)}$ can be obtained from solving Eq. (3.20). Similar analysis can be applied, and it is seen that the third-order and forth-order equations are respectively:

$$
\begin{gathered}
\phi_{(i)}^{(3)} \cdot u_{, x^{i}}^{(0)}+3 \phi_{(i)}^{(2)} \cdot u_{, x^{i}}^{(1)}+3 \phi_{(i)}^{(1)} \cdot u_{, x^{i}}^{(2)}+\phi_{(i)}^{(0)} \cdot u_{, x^{i}}^{(3)}=0 \\
\phi_{(i)}^{(4)} \cdot u_{, x^{i}}^{(0)}+4 \phi_{(i)}^{(3)} \cdot u_{, x^{i}}^{(1)}+6 \phi_{(i)}^{(2)} \cdot u_{, x^{i}}^{(2)}+4 \phi_{(i)}^{(1)} \cdot u_{, x^{i}}^{(3)}+\phi_{(i)}^{(0)} \cdot u_{, x^{i}}^{(4)}=0 .
\end{gathered}
$$


Finally, for the n'th-order, we have:

$$
\sum_{k=0}^{n} \frac{n !}{k !(n-k) !} \cdot \phi_{(i)}^{(n-k)} \cdot u_{, x^{i}}^{(k)}=0 .
$$

The solution of the (3.1) is:

$$
u=u^{(0)}+u^{(1)}+\frac{1}{2 !} u^{(2)}+\cdots .
$$

In the next section, we apply the above method for the Black-Scholes equation to price an European call option.

\section{Black-Scholes equation}

We consider the Black-Scholes equation (1.2) as:

$$
r u(x, t)-r x u_{, x}-\frac{\sigma^{2}}{2} x^{2} u_{, x x}=u_{, t} .
$$

In comparison with the Eq. (3.1), it is seen that

$$
\phi_{(0)}=r, \quad \phi_{(1)}=-r x, \quad \phi_{(2)}=-\frac{\sigma^{2}}{2} x^{2} .
$$

By considering $E(x)=x^{2}$, we have:

$$
\phi_{(0)}=\frac{r}{2} E_{, x x}, \quad \phi_{(1)}=-\frac{r}{2} E_{, x}, \quad \phi_{(2)}=-\frac{\sigma^{2}}{2} E .
$$

Using (2.7) and (2.8):

$$
\langle E\rangle=\int_{0}^{1} x^{2}=\frac{1}{3}
$$

and

$$
E^{\prime}=x^{2}-\frac{1}{3}
$$

According to $\phi^{(0)}=\left.\phi\right|_{E=<E>}$ and (4.3), we have:

$$
\phi_{(0)}^{(0)}=\frac{r}{2}(\langle E\rangle)_{, x x}=0, \quad \phi_{(1)}^{(0)}=-\frac{r}{2}(\langle E\rangle)_{, x}=0, \quad \phi_{(2)}^{(0)}=-\frac{\sigma^{2}}{2}\langle E\rangle=-\frac{\sigma^{2}}{6} .
$$

We consider Eq. (4.1) as:

$$
\phi_{(0)} u+\phi_{(1)} u_{, x}+\phi_{(2)} u_{, x x}=u_{, t} .
$$

For the first step, Eq. (4.7) can be written as:

$$
\phi_{(0)}^{(0)} u^{(0)}+\phi_{(1)}^{(0)} u_{, x}^{(0)}+\phi_{(2)}^{(0)} u_{, x x}^{(0)}=u_{, t}^{(0)} .
$$

So by Eq. (4.6) we have

$$
-\frac{\sigma^{2}}{6} u_{, x x}^{(0)}=u_{, t}^{(0)}
$$


We should solve Eq. (4.9) with boundary and terminal conditions (1.3), (1.4) and (1.5). By using $\tau=T-t$, Eq. (4.9) and its conditions will be:

$$
\frac{\sigma^{2}}{6} \nu_{, x x}^{(0)}=\nu_{, \tau}^{(0)}
$$

with

$$
\nu^{(0)}(x, 0)=\max (x-K, 0), \quad \nu^{(0)}(0, \tau)=0, \quad \nu^{(0)}(L, \tau)=L,
$$

which $L$ is adequate large. By considering $\nu^{(0)}(x, \tau)=V(x, \tau)+W(x)$ so that

$$
V(0, \tau)=0, \quad V(L, \tau)=0,
$$

we find $W(x)=x$. Therefore, instead of solving Eq. (4.10) subject to (4.11), we solve:

$$
\frac{\sigma^{2}}{6} V_{, x x}=V_{, \tau}
$$

with

$$
V(x, 0)=-x+\max (x-K, 0), \quad V(0, \tau)=0, \quad V(L, \tau)=0
$$

Consider

$$
V(x, \tau)=A(x) B(\tau) .
$$

By substituting (4.15) in Eq. (4.13), we have:

$$
\frac{B^{\prime}(\tau)}{B(\tau)}=\frac{\sigma^{2}}{6} \frac{A^{\prime \prime}(x)}{A(x)}=k,
$$

considering $k=-\lambda^{2}$ :

$$
\frac{B^{\prime}(\tau)}{B(\tau)}=-\lambda^{2}, \quad \frac{\sigma^{2}}{6} \frac{A^{\prime \prime}(x)}{A(x)}=-\lambda^{2}
$$

Therefore

$$
B(\tau)=d e^{-\lambda^{2} \tau}, \quad A(x)=c_{1} \sin \left(\frac{\sqrt{6} \lambda}{\sigma} x\right)+c_{2} \cos \left(\frac{\sqrt{6} \lambda}{\sigma} x\right) .
$$

Till here we have

$$
V(x, \tau)=\left(k_{1} \sin \left(\frac{\sqrt{6} \lambda}{\sigma} x\right)+k_{2} \cos \left(\frac{\sqrt{6} \lambda}{\sigma} x\right)\right) e^{-\lambda^{2} \tau},
$$

using boundary condition $V(0, \tau)=0$, we obtain $k_{2}=0$, and using boundary condition $V(L, \tau)=0$, we have:

$$
k_{1} \sin \left(\frac{\sqrt{6} \lambda}{\sigma} L\right) e^{-\lambda^{2} \tau}=0 .
$$

Since $e^{-\lambda^{2} \tau} \neq 0$ and $k_{1} \neq 0$, so $\sin \left(\frac{\sqrt{6} \lambda}{\sigma} L\right)=0$, and then $\lambda=\frac{\sigma}{\sqrt{6}} \frac{n \pi}{L}$, Therefore

$$
V(x, \tau)=k_{1} \sin \left(\frac{n \pi}{L} x\right) e^{-\frac{\sigma^{2}}{6} \frac{n^{2} \pi^{2}}{L^{2}} \tau} .
$$

Any linear combination of (4.21) is a solution of Eq. (4.13), so:

$$
V(x, \tau)=\sum_{n=1}^{\infty} k_{n} \sin \left(\frac{n \pi}{L} x\right) e^{-\frac{\sigma^{2}}{6} \frac{n^{2} \pi^{2}}{L^{2}} \tau} .
$$


By using initial condition $V(x, 0)=-x+\max (x-K, 0)$, we have

$$
-x+\max (x-K, 0)=\sum_{n=1}^{\infty} k_{n} \sin \left(\frac{n \pi}{L} x\right) .
$$

$k_{n}$ in (4.23) is the Fourier Sine series coefficients in $[0, L]$ :

$$
k_{n}=\frac{2}{L} \int_{0}^{L}(-x+\max (x-K, 0)) \sin \left(\frac{n \pi}{L} x\right) d x .
$$

So

$$
\nu^{(0)}(x, \tau)=x+\sum_{n=1}^{\infty} k_{n} \sin \left(\frac{n \pi}{L} x\right) e^{-\frac{\sigma^{2}}{6} \frac{n^{2} \pi^{2}}{L^{2}} \tau}
$$

and then

$$
u^{(0)}(x, t)=x+\sum_{n=1}^{\infty} k_{n} \sin \left(\frac{n \pi}{L} x\right) e^{-\frac{\sigma^{2}}{6} \frac{n^{2} \pi^{2}}{L^{2}}(T-t)}
$$

For $u^{(1)}$ we have

$$
\phi_{(0)}^{(0)} u^{(1)}+\phi_{(1)}^{(0)} u_{, x}^{(1)}+\phi_{(2)}^{(0)} u_{, x x}^{(1)}+\phi_{(0)}^{(1)} u^{(0)}+\phi_{(1)}^{(1)} u_{, x}^{(0)}+\phi_{(2)}^{(1)} u_{, x x}^{(0)}=u_{, t}^{(1)} .
$$

On the other hand, $\phi_{(0)}^{(1)}$ is obtained as:

$$
\phi_{(0)}^{(1)}=\phi_{(0), E_{1}} * E_{1}^{\prime}=\frac{r}{2} E_{, x x E_{1}} * E_{1}^{\prime}=\frac{r}{2} \delta_{, x x} * E_{1}^{\prime}=\frac{r}{2} E_{, x x}^{\prime},
$$

(2.3) and (2.12) have been used respectively for the last two equalities in (4.28). Similarly, $\phi_{(1)}^{(1)}$ and $\phi_{(2)}^{(1)}$ are obtained as:

$$
\begin{aligned}
& \phi_{(1)}^{(1)}=\phi_{(1), E_{1}} * E_{1}^{\prime}=-\frac{r}{2} E_{, x E_{1}} * E_{1}^{\prime}=-\frac{r}{2} \delta_{, x} * E_{1}^{\prime}=-\frac{r}{2} E_{, x}^{\prime}, \\
& \phi_{(2)}^{(1)}=\phi_{(2), E_{1}} * E_{1}^{\prime}=-\frac{\sigma^{2}}{2} E_{, E_{1}} * E_{1}^{\prime}=-\frac{\sigma^{2}}{2} \delta * E_{1}^{\prime}=-\frac{\sigma^{2}}{2} E^{\prime} .
\end{aligned}
$$

So we have:

$$
\begin{gathered}
\phi_{(0)}^{(1)}=\frac{r}{2}\left(x^{2}-\frac{1}{3}\right)_{, x x}=r, \\
\phi_{(1)}^{(1)}=-\frac{r}{2}\left(x^{2}-\frac{1}{3}\right)_{, x}=-r x, \\
\phi_{(2)}^{(1)}=-\frac{\sigma^{2}}{2}\left(x^{2}-\frac{1}{3}\right),
\end{gathered}
$$

and then Eq. (4.27) is:

$$
-\frac{\sigma^{2}}{6} u_{, x x}^{(1)}+r u^{(0)}-r x u_{, x}^{(0)}-\frac{\sigma^{2}}{2}\left(x^{2}-\frac{1}{3}\right) u_{, x x}^{(0)}=u_{, t}^{(1)} .
$$

Now, by substituting (4.26) and its derivatives in Eq. (4.34) we have

$$
-\frac{\sigma^{2}}{6} u_{, x x}^{(1)}=u_{, t}^{(1)}+F(x, t)
$$


which $F(x, t)=-r u^{(0)}+r x u_{, x}^{(0)}+\frac{\sigma^{2}}{2}\left(x^{2}-\frac{1}{3}\right) u_{, x x}^{(0)}$. In this step, we use homogeneous conditions:

$$
u^{(1)}(x, T)=0, \quad u^{(1)}(0, t)=0, \quad u^{(1)}(L, t)=0 .
$$

As we can see, Eq. (4.35) differs from Eq. (4.9) by the $F(x, t)$ only. Generally, more terms of Frechet series, by the FPM procedures obtain from recursive differential equations:

$$
-\frac{\sigma^{2}}{2}\langle E\rangle u_{, x x}^{(k)}+\frac{r}{2} E_{, x x}^{\prime} u^{(k-1)}-\frac{r}{2} E_{, x}^{\prime} u_{, x}^{(k-1)}-\frac{\sigma^{2}}{2} E^{\prime} u_{, x x}^{(k-1)}=u_{, t}^{(k)} \quad k \geq 1,
$$

with homogeneous terminal and boundary conditions. Finally, the solution of Eq. (4.1) is:

$$
u=u^{(0)}+u^{(1)}+\frac{1}{2 !} u^{(2)}+\cdots .
$$

\section{Conclusions}

To sum up, this paper considers a powerful mathematical tool based on functional perturbation method to price the European call option of Black-Scholes equation. The FPM is an explicit method that deals with Frechet series of functions, leading to a set of partial differential equations with constant coefficients. We do not need to resolve a new partial differential equation for each step. All equations differ only in their right hand side parts. The authors believe that this method can be extended to price an American option.

\section{REFERENCES}

[1] E. Altus. Analysis of Bernoulli beams with 3D stochastic hetrogeneity Probabilist. Eng. Mech., 18: 301$314,2003$.

[2] E. Altus. Microstress estimate of stochastically hetrogeneous structures by the functional perturbation method: A one dimentional example, Probabilist. Eng. Mech., 21: 434-441, 2006.

[3] E. Altus, Statistical modeling of heterogeneous microbeams. Int. J. Solids. Struct., 38: 5915-5034, 2001.

[4] E. Altus and E. Totry, Buckling of stochastically heterogeneous beams using a functional perturbation method, Int. J. Solids. Struct., 40: 6547-6565, 2003.

[5] E. Altus. A. Proskura and S. Givli, A new functional perturbation method for linear non-homogeneous materials. Int. J. Solids. Struct., 42: 1577-1595, 2005.

[6] E. Altus. E. Totry and S. Givli, Optimized functional perturbation method and morphology based effective properties of randomly heterogeneous beams, Int. J. Solids. Struct., 42: 2345-2359, 2005

[7] M. Avellaneda and A. Paras, Dynamic hedging portfolios for derivative securities in the presence of large transaction costs, Appl. Math. Finance., 1: 165-193, 1994.

[8] B. Bensaid. J. Lesne. H. Pages and J. Scheinkman, Derivative asset pricing with transaction costs, Math. Finance., 2: 63-82, 1992.

[9] M. Beran, Statistical continuum mechanics, Inter-Science Publishers, 1968.

[10] F. Black and M. Scholes, The pricing of options and corporate liabilities, J. Polit. Econ., 81: 637-54, 1973.

[11] P. Boyle and T. Vorst, Option replication in discrete time with transaction costs, J. Finance., 47: 271-293, 1973.

[12] M. Dehghan and S. Pourghanbar, Solution of the Black-Scholes Equation for Pricing of Barrier Option, Z Naturforsch., 66a: 289-296, 2011.

[13] S. Givli and E. Altus, Improved functional perturbation method for reliability analysis of randomly heterogeneous beams, Struct. Saf., 28: 378-391, 2006.

[14] H.C. Hull, Fundamentals of Futures and Options Markets, Fourth edition, Prentice Hall, 2002.

[15] R.P. Kanwal, Generalized functions: Theary and techniques, Birkhauser, Basel, 1998.

[16] S. Nachum and E. Altus. Natural frequenvies and mode shapes of deterministic and stochastic non homogeneous rods and beams, J. Sound. Vib., 302: 903-924, 2007.

[17] B. Oksendal, Stochastic differential Equations: An Introduction with Applications, Sixth Edition, Springer, 2000 . 
[18] S. Poon, A Practical Guide to Forecasting financial market volatility, John Wiley \& Sons Ltd. 2005.

[19] S.A. Ramu and R. Ganesan, Response and stability of stochastic beam column using stochastic FEM, Comp. Struct., 54: 207-221, 1994.

[20] E.M. Totry. E. Altus. and A. Proskura, A novel application of the FPM to the buckling differential equation of non uniform beams, Probabilist. Eng. Mech., bf 23: 339-346, 2008.

[21] E. Totry. E. Altus. and A. Proskura, Buckling of non uniform beams by a direct functional perturbation method, Probabilist. Eng. Mech., 22: 88-99, 2007.

[22] P. Wilmott. S. Howison and J. Dewynne, The Mathematics of Financial Derivatives, Cambridge Univ Press, Cambridge, 1995.

[23] P. Wilmott, Introduces quantitative finance, Second Edition, John Wiley \& sons, 2007.

[24] P. Wilmott. J. Dewynne and S. Howison, Option pricing mathematical models and computation, Oxford Financial Press, Oxford, 1993.

(Somayeh Pourghanbar) Department of Mathematics, Azarbaijan shahid madani University, Tabriz, IRAN.

Email address: s.pourghanbar@aut.ac.ir \& somayepourghanbar@gmail.com

(Mojtaba Ranjbar) Department of Mathematics, Azarbaijan shahid madani University, Tabriz, IRAN.

Email address: m_ranjbar@azaruniv.ac.ir

(Ebrahim Nasrabadi) Department of Mathematics, University of Birjand, Birjand, Iran.

Email address: nasrabadi@birjand.ac.ir 